\title{
ANALYSIS OF LINKS POSITIONS IN LANDING GEAR MECHANISM
}

\author{
D. BREWCZYNSSKI ${ }^{*}$ and G. TORA \\ Cracow University of Technology \\ Faculty of Mechanical Engineering \\ Institute of Machine Design \\ Jana Pawla II 37, 31-864 Cracow, POLAND \\ E-mails: brewczyn@mech.pk.edu.pl; tora@mech.pk.edu.pl
}

\begin{abstract}
This article contains a kinematic analysis of an aircraft chassis mechanism in a range of positions. The mechanism of the chassis is made up of several smaller subsystems with different functions. The first mechanism is used to eject the chassis before landing (touchdown) and fold it to hatchway after the lift off. The second mechanism is designed to perform rotation of the crossover with the wheel, in order to adjust the position of the wheel to fit it in the limited space in the hold. The third mechanism allows movement of the chassis resulting from the change in length of the damper. To determine the position of the following links of the mechanism calculus of vectors was applied in which unit vectors were used to represent the angular position of the links. The aim of the analysis is to determine the angle of convergence and the angle of heel wheels as a function of the variable length of hydraulic cylinder, length of the shock absorber, length of the regulations rods.
\end{abstract}

Key words: aircrafts, landing gear, mechanics, kinematics.

\section{Introduction}

A typical solution in the construction of an aircraft flight decreasing aerodynamic drag is the mechanism comprising a movable chassis to hatch located in the body of the aircraft. In the aircraft Lockheed F-104S Starfighter, a movable main landing gear is located in the central part of the fuselage.

The paper contains a kinematic analysis of the spatial mechanism chassis (Fig.1) in a range of positions. The analysis determined the convergence angle and the angle of heel landing wheels as a function of chassis extendible hydraulic cylinder length, the length of the shock absorber and two adjustable links. The analysis was performed applying a vector method using versors representing the angular position of the links (Chace, 1965; Shigley and Uicker, 1995).

\section{Construction of the landing gear mechanism}

A gear mechanism consists of three smaller mechanisms that perform different functions (Brewczyński and Tora, 2013). The first mechanism, (Fig.2) is used to eject the landing gear before landing and fold it into the hold after liftoff. It consists of two fixed-length links 2 and 3 and the hydraulic cylinder 4, connected to a double pivot $D$. The shank 1 is connected to actuator 4 by pivot $E$ and to link 2 by pivot $C$. To connect link 3 to the airframe were used movable link 10 was used.

Link 10 is connected with link 3 by pivot $B$ and with airplane frame by pivot $N$. Rotary joint axes $A$, $B, C, D$ and $E$ are parallel to each other, so that a part of the chassis, composed of links 3, 2, shank 1 and actuator 4 can be regarded as a flat mechanism (Fig.2).

\footnotetext{
* To whom correspondence should be addressed
} 


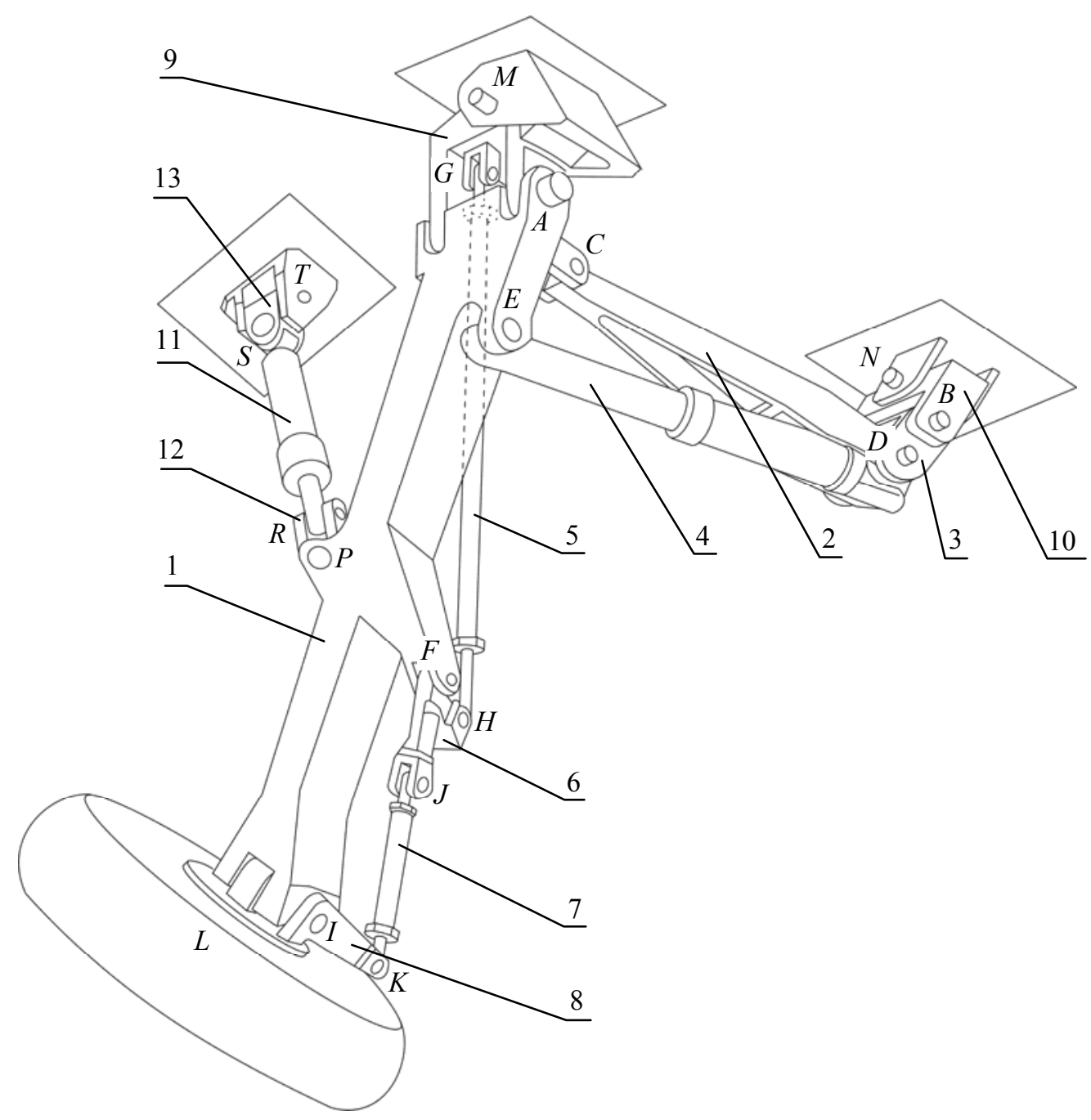

Fig.1. The mechanism of the aircraft landing gear.

The second gear mechanism (Fig.3) is designed to perform a limited rotation of the steering 8 with the wheel around pivot $I$ caused by movement of the shank 1 . The rotation around the axis of pivot $I$ should provide accurate positioning of the wheel in two extreme positions. The first results from the necessity of such a setting that wheel will be able to fold and fit in to the expected area of the chassis hatch. The second extreme position refers to the total distribution of the chassis, where the wheel should achieve established angle to the shank. Rotation of the wheel steering joint $I$ is forced by the space arrangement of two quadrangle, connected to the shank. The first quadrangle is made up of connector 9 , shank 1 , connector 5 and wishbone 6 . Wishbone 6 is connected to the shank by pivot $F$. Link 5 is connected to the connector 9 and the wishbone 6 by ball joints $G$ and $H$. The second quadrangle consists of wishbone 6 , shank 1 , connector 7 and steering 8 . The steering 8 is connected to the shank by joint $I$. Link 7 is connected to the wishbone 6 and steering 8 by ball joints $J$ and $K$. The main landing gear wheel is mounted on the pivot 8 in the rotary joint $L$. Changing the length of the actuator 4 movable landing gear mechanism rotates the main shank 1 around the pivot $A$ and simultaneously the steering 8 wheel rotates around pivot axis $I$. In the mechanism of movable chassis connectors 5 and 7 have adjustable lengths. The use of such regulation compensates for inaccuracies of links performance and their assembly to ensure accurate positioning of the wheels in the extreme positions. 


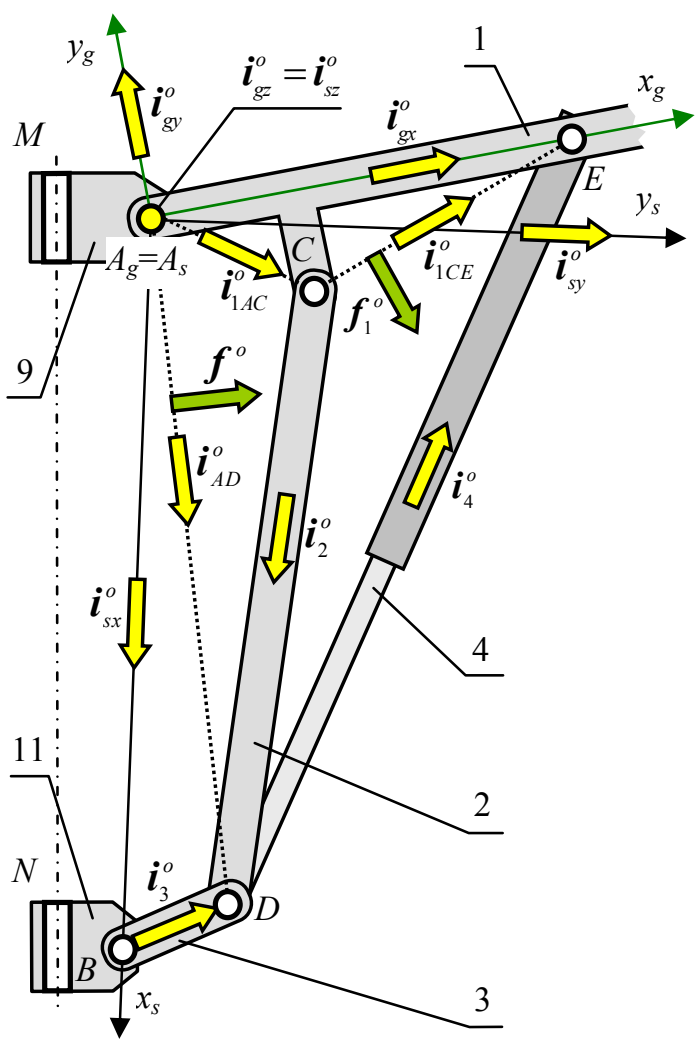

Fig.2. Chassis eject mechanizm.

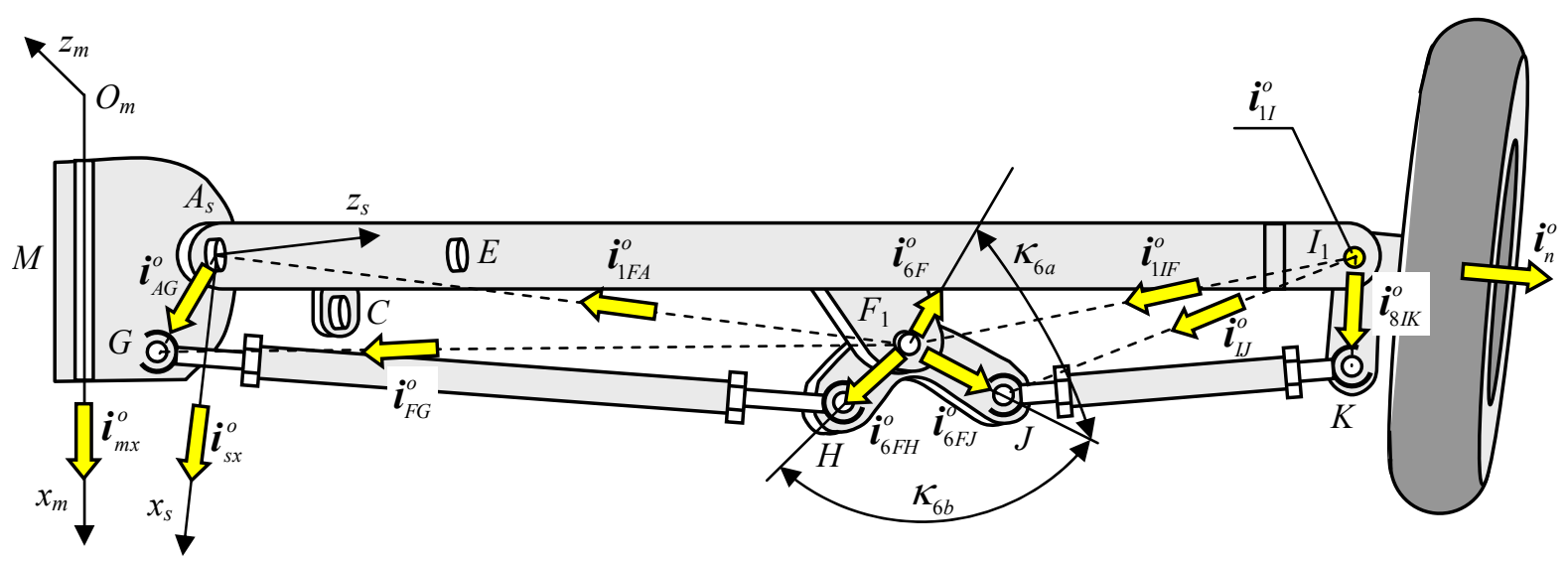

Fig.3. The chassis mechanism that causes rotation of the steering wheel.

The third mechanism (Fig.4) allows movement of the chassis resulting from a change in the length of the elastic-damping (hereinafter referred to as a shock absorber) 11 . The shank is connected indirectly to the airframe by a movable connector 9 , connected to the plane frame by pivot $M$ and to the shank by pivot $A$. Shock absorber 11 is positioned over the shank connected thereto and to the plane frame by means of connectors 12 and 13. Connector 12 is connected to the shank 1 by pivot $P$, and to the shock absorber by pivot $R$. Connector 13 is connected to the frame pivot plane $T$, and shock absorber pivot $S$. During take-off and landing the aircraft, as a result of changes in the length of the shank damper rotates around a pivot axis 
$M$. Joints $M$ and $N$ have a common axis of rotation which is parallel to the line of intersection of the plane symmetry and the surface on which the aircraft is.

The landing gear mechanism cooperating with the opening and closing mechanisms of the two flaps. These mechanisms are not shown in Fig. 1 and are not considered in this study.

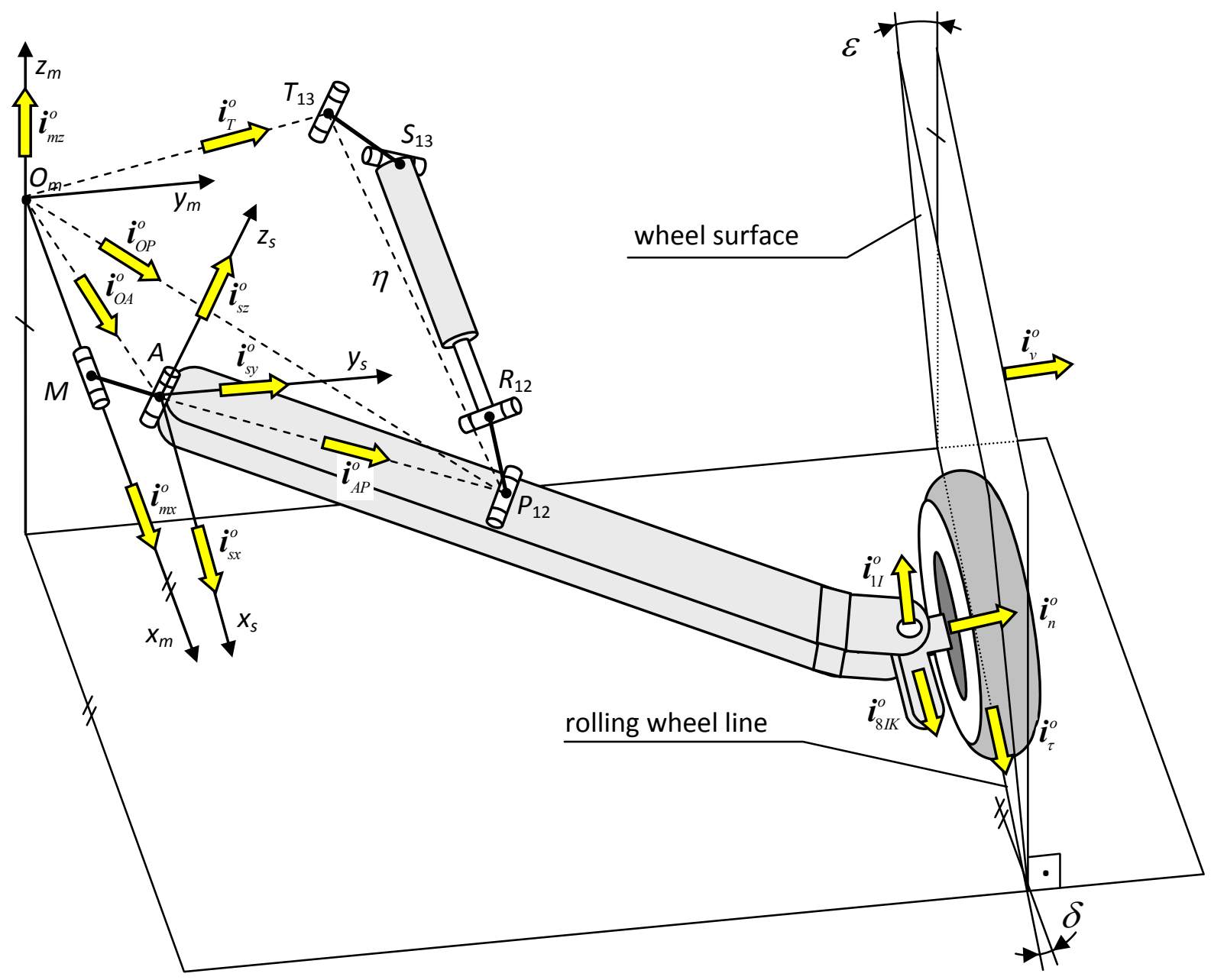

Fig.4. The mechanism with shock absorber.

\section{Analysis of links positions in the landing gear mechanism}

To determine the positions of the chassis links five types of equations were used repeatedly. The sequence numbers used in the schemes of calculations are presented in Figs 5, 6 and 7.

1) Determine the unit vector $\boldsymbol{x}^{o}$ perpendicular to another known unit vector $\boldsymbol{y}^{o}$, both are on the plane perpendicular to the unit vector $\boldsymbol{z}^{o}: \boldsymbol{x}^{o}=\boldsymbol{y}^{o} \times \boldsymbol{z}^{o}$.

2) Determine unit vectors $\boldsymbol{u}_{i}^{o}$ and $\boldsymbol{u}_{j}^{o}$ which satisfy the relations: $u_{i} \boldsymbol{u}_{i}^{o}+u_{j} \boldsymbol{u}_{j}^{o}=u_{k} \boldsymbol{u}_{k}^{o}$, if the modules $u_{i}$, $u_{j}, u_{k}$ and unit vector $\boldsymbol{u}_{k}^{o}$ are known:

$$
\boldsymbol{u}_{i}^{o}= \pm \sqrt{1-\left(\frac{u_{i}^{2}-u_{j}^{2}+u_{k}^{2}}{2 u_{k} u_{i}}\right)^{2}} \boldsymbol{f}^{o}+\frac{u_{i}^{2}-u_{j}^{2}+u_{k}^{2}}{2 u_{k} u_{i}} \boldsymbol{u}_{k}^{o}
$$




$$
\boldsymbol{u}_{j}^{o}=\mp \sqrt{\left(\frac{u_{i}}{u_{j}}\right)^{2}-\left(\frac{u_{i}^{2}-u_{j}^{2}+u_{k}^{2}}{2 u_{k} u_{j}}\right)^{2}} \boldsymbol{f}^{o}+\frac{u_{j}^{2}-u_{i}^{2}+u_{k}^{2}}{2 u_{k} u_{j}} \boldsymbol{u}_{k}^{o}
$$

where: $\boldsymbol{f}^{o}$ - unit vector perpendicular to $\boldsymbol{u}_{k}^{o}$.

3) Determine the unit vector $\boldsymbol{u}_{k}^{o}$ from equation: $u_{i} \boldsymbol{u}_{i}^{o}+u_{j} \boldsymbol{u}_{j}^{o}=u_{k} \boldsymbol{u}_{k}^{o}$, if the modules $u_{i}, u_{j}, u_{k}$ are known and unit vectors: $\boldsymbol{u}_{i}^{o}$ and $\boldsymbol{u}_{j}^{o}: \boldsymbol{u}_{k}^{o}=\frac{u_{i} \boldsymbol{u}_{i}^{o}+u_{j} \boldsymbol{u}_{j}^{o}}{u_{k}}$.

4) Determine the unit vector $\boldsymbol{a}^{o}$ perpendicular to two other known $\boldsymbol{c}^{o}$ and $\boldsymbol{b}^{o}: \boldsymbol{a}^{o}=\frac{\boldsymbol{b}^{o} \times \boldsymbol{c}^{o}}{\left\|\boldsymbol{b}^{o} \times \boldsymbol{c}^{o}\right\|}$.

5) Determine the unit vector $\boldsymbol{w}_{k}^{o}$ on the basis of two known unit vectors $\boldsymbol{w}_{i}^{o}, \boldsymbol{w}_{j}^{o}$ and three cosines of the angles between all unit vectors $c_{i k}, c_{j k}, c_{i j}$

$$
\boldsymbol{w}_{k}^{o}=\frac{c_{i k}-c_{i j} c_{j k}}{1-c_{i j}^{2}} \boldsymbol{w}_{i}^{o}+\frac{c_{j k}-c_{i j} c_{i k}}{1-c_{i j}^{2}} \boldsymbol{w}_{j}^{o} \pm \frac{\sqrt{1-c_{i j}^{2}-c_{i k}^{2}-c_{j k}^{2}+2 c_{i j} c_{i k} c_{j k}}}{1-c_{i j}^{2}}\left(\boldsymbol{w}_{i}^{o} \times \boldsymbol{w}_{j}^{o}\right)
$$

6) Determine angle $\alpha$ between unit vectors $\boldsymbol{d}^{o}$ and $\boldsymbol{e}^{o}: \alpha=\operatorname{sgn}\left[\boldsymbol{h}^{o} \cdot\left(\boldsymbol{d}^{o} \times \boldsymbol{e}^{o}\right)\right] \arcsin \left(\left|\boldsymbol{d}^{o} \times \boldsymbol{e}^{o}\right|\right)$, where: $\boldsymbol{h}^{o}$ - unit vector relative to which the sign of the angle is determined.

Each of the three mechanisms is described in their own reference systems in which they determined the position of the links. The first reference system $\left\{A_{g} x_{g} y_{g} z_{g}\right\}$ is related to the shank 1 (Fig.2). The axis $z_{g}$ lies in the pivot axis $A$. The axis $x_{g}$ intersects at a straight angle joint axes $\mathrm{A}$ and $\mathrm{E}$. The center $A_{g}$ is the point of intersection of the pivot axis $A$ and perpendicular to the surface containing the center of the ball joint $G$.

The second reference system $\left\{A_{s} x_{s} y_{s} z_{s}\right\}$ is related to switches 9 and 10, which perform rotation around a common pivot axis $M$ and $N$ (Figs 2 and 3). The axis $z_{s}$ coincides with the axis $z_{g}$ and $x_{g}$ axis intersects a perpendicular pivot axes $A$ and $E$. Points $A_{s}$ and $A_{g}$ overlap. In this reference system displacements of the wheel orienting mechanism links are calculated.

The third reference system $\left\{O_{m} x_{m} y_{m} z_{m}\right\}$ is related to the plane frame (Figs 3 and 4). The axis $x_{m}$ lies on a common pivot axis $M$ and $N$. The axis $z_{m}$ is perpendicular to the surface on which the aircraft stands. $O_{m}$ is the point of intersection of the plane $z_{m} y_{m}$ with the axis $x_{m}$. The third reference system allows calculation of the taper angle $\delta$ resulting from the change in the length of the shock-absorber

$$
\delta=\operatorname{sgn}\left[{ }^{m} \boldsymbol{i}_{m x}^{o} \cdot\left({ }^{m} \boldsymbol{i}_{\tau}^{o} \times{ }^{m} \boldsymbol{i}_{m x}^{o}\right)\right] \arcsin \left\|{ }^{m} \boldsymbol{i}_{\tau}^{o} \times{ }^{m} \boldsymbol{i}_{m x}^{o}\right\|,
$$

and the tilt angle of the wheel $\varepsilon$ (Reimpell and Betzler, 2004)

$$
\varepsilon=\operatorname{sgn}\left[{ }^{m} \boldsymbol{i}_{\tau}^{o} \cdot\left({ }^{m} \boldsymbol{i}_{v}^{o} \times{ }^{m} \boldsymbol{i}_{n}^{o}\right)\right] \arcsin \left\|{ }^{m} \boldsymbol{i}_{v}^{o} \times{ }^{m} \boldsymbol{i}_{n}^{o}\right\|
$$

\section{Calculation algorithms}

Wheel orientation angles (3.1), (3.2) are determined based on unit vectors expressed in the reference system $\left\{O_{m} x_{m} y_{m} z_{m}\right\}$. Other unit vector calculations are carried out in three different reference systems 
$\left\{A_{g} x_{g} y_{g} z_{g}\right\},\left\{A_{s} x_{s} y_{s} z_{s}\right\}$ and $\left\{O_{m} x_{m} y_{m} z_{m}\right\}$. It is therefore necessary to create two transition matrixes: ${ }_{g}^{s} \boldsymbol{R}$ and ${ }_{s}^{m} \boldsymbol{R}$.

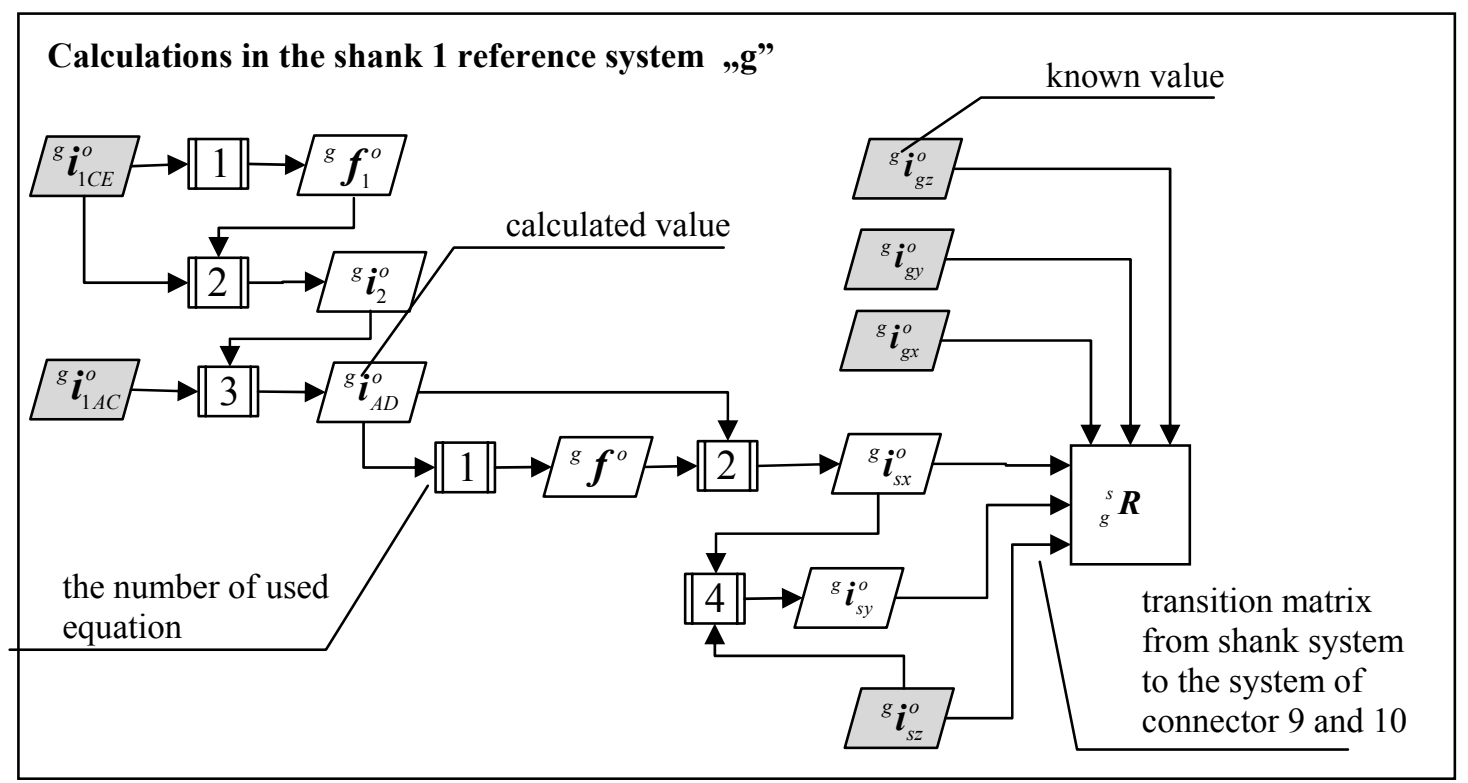

Fig.5. The calculation algorithm in the shank reference system.

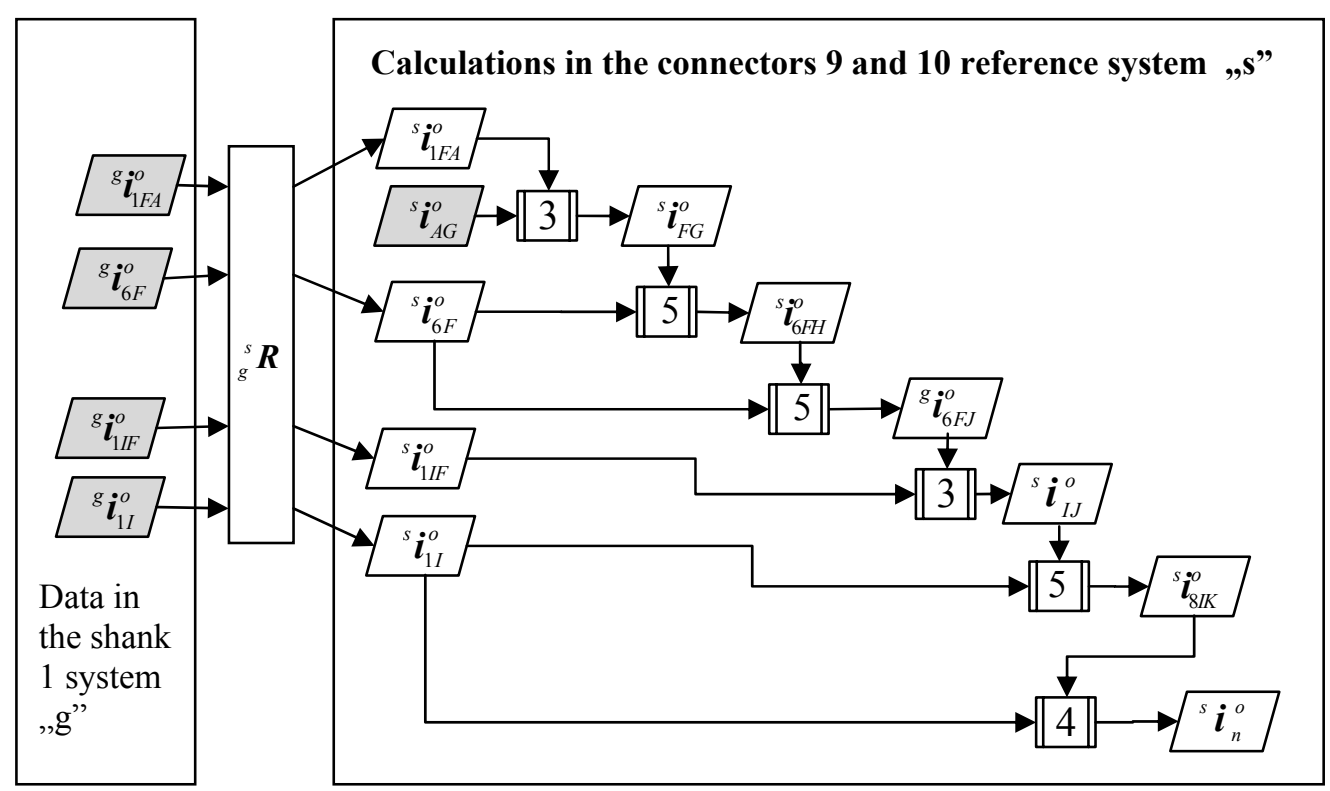

Fig.6. The calculation algorithm in the connector 9 and 10 reference system. 


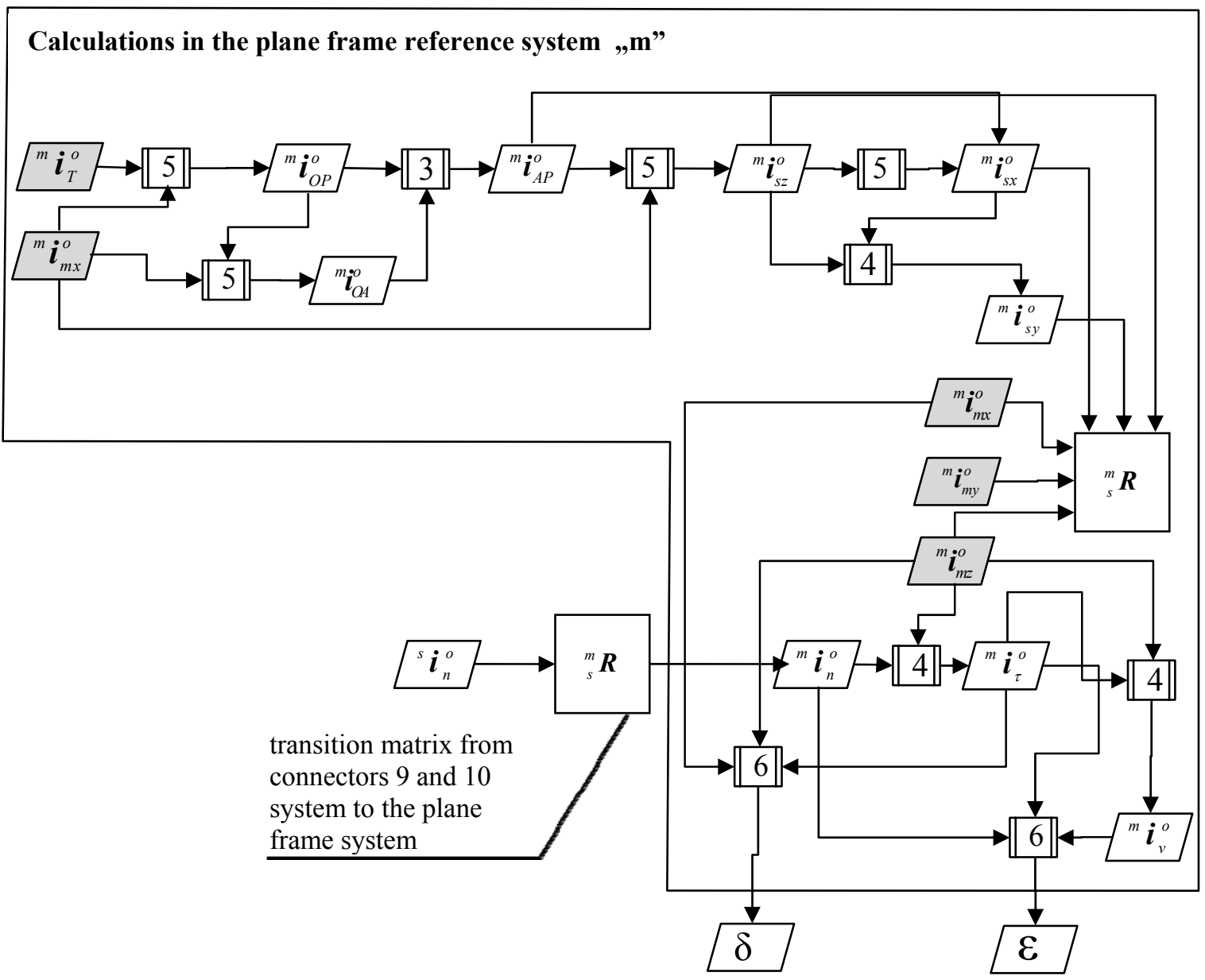

Fig.7. The calculation algorithm in the plane frame reference system.

\section{Results and conclusions}

The landing gear mechanism is an example of the usefulness of analysis of links in several different reference systems. It requires the designation of the appropriate transition matrix. The analysis can be the basis to examine the impact of accuracy of performance and links assembly mechanism on angular position of the wheel chassis.

It has been shown that by changing the length of the shock absorber of the landing gear wheel, the taper angle changes slightly while the wheels tilt angle changes in the range $\left(9.2^{\circ} \div-6.0^{\circ}\right)$.

It has been shown that for a range of length variation of links 5 and 7 the taper angle $\delta$ (Fig.9) changes in the range $\left(-12.14^{\circ} \div 11.13^{\circ}\right)$ and the tilt angle remains the same. 


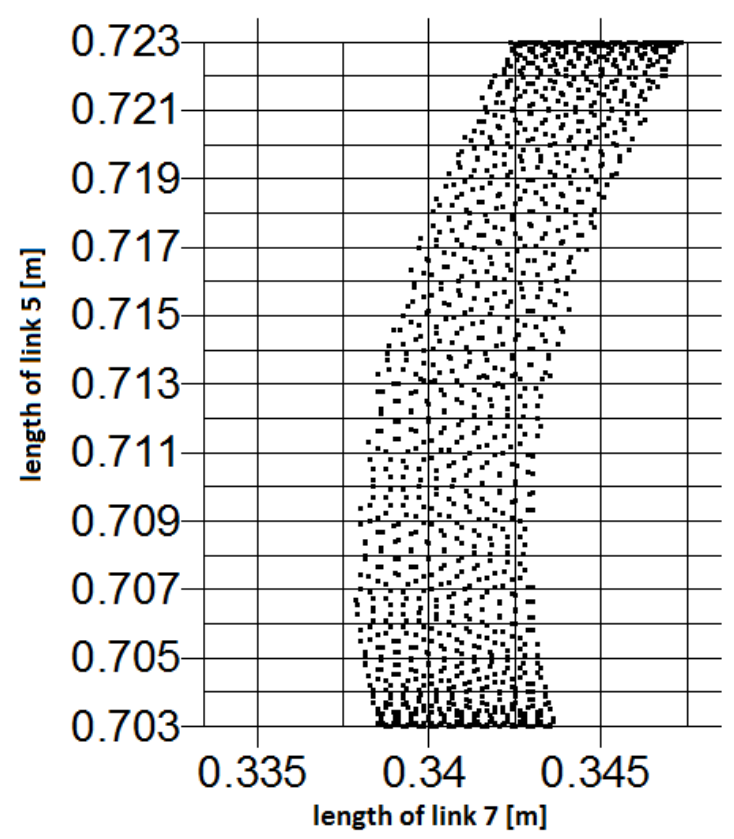

Fig.8. Lengths of links 5 and 7 which give the taper angle in a proper range $\left[-1^{\circ}, 1^{\circ}\right]$.

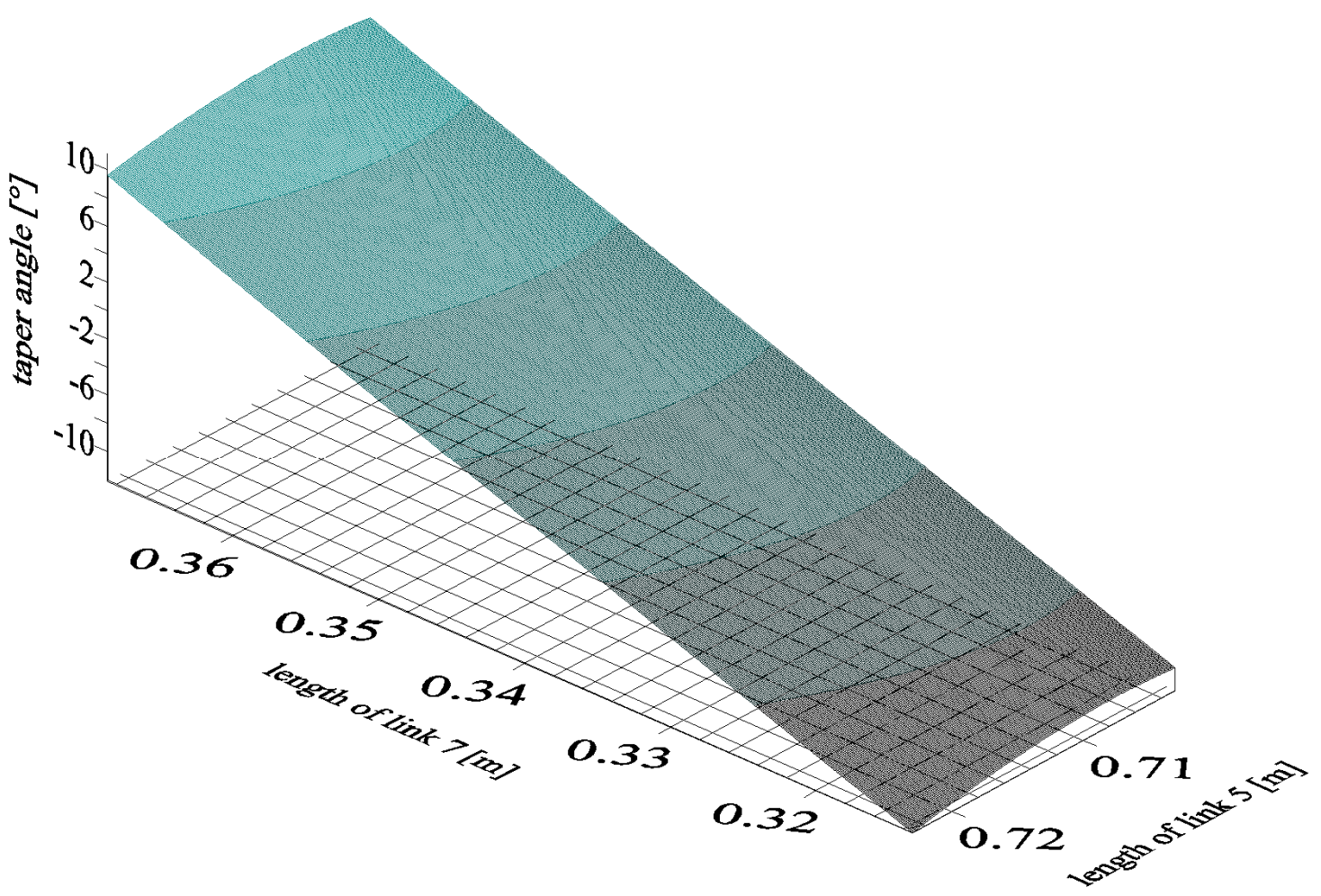

Fig.9. Taper angle of wheel as a function of lengths of links 5 and 7. 


\section{Nomenclature}

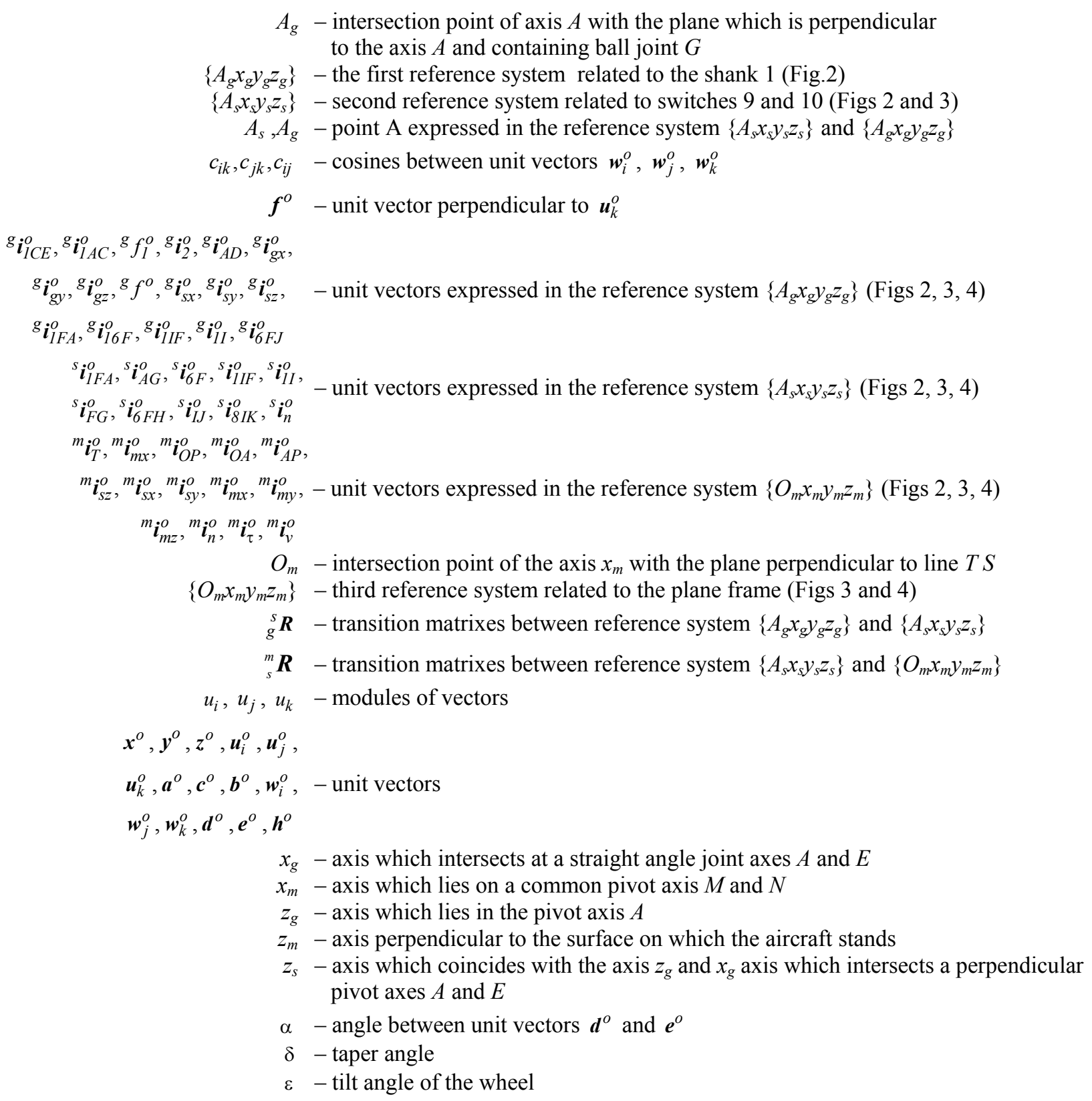

\section{References}

Brewczyński D. and Tora G. (2013): Analysis of the wheel orientation angles in Lockheed F-104S starfighter aircraft. Journal of Kones, vol.20, No.3, pp.79-87.

Cervantes-Sanchez J.J., Moreno-Baez M.A., Aguilera-Cortes L.A. and Gonzalez-Galvan E.J. (2005): Kinematic design of the RSSR-SC spatial linkage based on rotability conditions. - Mechanism and Machine Theory, vol.40, pp.11261144.

Chace M.A. (1965): Solutions to the vector tetrahedron equation. - Transactions of the ASME, Journal of Engineering for Industry, vol.87, Series B, pp.228-234. 
Manuale tecnico catalogo nomenclatore illustrato delle parti di ricambio VELIVOLO F-104S.

Psang Dain Lin and Jung-Fa Hsieh (2007): A new method to analyze spatial binary mechanisms with spherical pairs.Journal of Mechanical Design, vol.129, pp.455-458.

Reimpell J. and Betzler J. (2004): Car chassis. Basics of structures. - Warsaw: WKit.

Shigley J.E. and Uicker J.J. (1995): Theory of Machines and Mechanisms. - McGraw-Hill Book.

Received: May 13, 2014

Revised: June 17, 2014 